\title{
On the Impact of Antenna Correlation on the Pilot-Data Balance in Multiple Antenna Systems
}

\author{
Gábor Fodor \\ Ericsson Research \\ Gabor.Fodordericsson.com \\ and \\ Royal Institute of Technology \\ gaborfakth.se
}

\author{
Miklós Telek \\ Budapest Univ. of Technology and Economics \\ telek@hit.bme.hu
}

\author{
Piergiuseppe di Marco \\ Royal Institute of Technology \\ pidmakth.se
}

\begin{abstract}
We consider the uplink of a single cell single input multiple output (SIMO) system, in which the mobile stations use intra-cell orthogonal pilots to facilitate uplink channel estimation. In such systems, the problem of pilot-data transmission power balancing is known to have a large impact on the performance on the achievable uplink data rates. In this paper we derive a closed form expression for the mean square error (MSE) as a function of the pilot and data power levels under a per-user sum pilot-data power constraint. Our major contribution is the derivation of the MSE formula for Gaussian channels under arbitrary channel covariance matrices. For example, our model readily allows to study the MSE as a function of the number of antennas and antenna correlation structures, including the popular spatial channel model (SCM). Numerical results suggest that the impact of antenna spacing on the MSE is limited, but the angle of arrival (AoA) and angular spread have a more articulated impact on the MSE performance. We also find that as the number of antennas at the base station grows large, a higher percentage of the power budget should be allocated to pilot signals than with a low number of antennas.
\end{abstract}

\section{INTRODUCTION}

In multiple input multiple output (MIMO) orthogonal frequency division multiplexing (OFDM) systems pilot symbols facilitate channel estimation, but they reduce the transmitted energy for data symbols under a fixed power budget. This fundamental tradeoff has been studied in [1] and [2] assuming various pilot patterns and receiver structures. An important insight from these works is that although balancing the pilotto-data power ratio (PDPR) improves the system performance, the capacity is not highly sensitive to the PDPR over a fairly broad range. The results of [2], for example, indicate that the optimal PDPR provides about 2-3 dB gain compared with the equal power for pilot and data symbols.

Subsequently, [3] derived a closed form of the optimal PDPR in MIMO-OFDM spatial multiplexing systems with minimum mean square error channel estimation and showed that a tight bound lying in the quasi-optimal region provides a good approximation for the optimal PDPR setting. More recently, [4] derived a closed form PDPR that maximizes the capacity bound of MIMO-OFDM systems and studied the impact of carrier frequency offset (CFO) on the maximizing power allocation.

In previous works we considered the case of an isolated cell with uncorrelated antennas giving rise to a diagonal covariance matrix [5]. The multicell case with diagonal covariance matrix was considered in [6], where it was found that the so called distributed iterative channel inversion (DICI) algorithm originally proposed by [7] can be advantageously extended taking into account the pilot-data power trade off. However, none of these works capture the impact of antenna correlation on the performance of single input multiple output (SIMO) systems.

In this paper we consider a SIMO system in which the mobile station balances its PDPR, while the base station uses maximum likelihood (ML) channel estimation to initialize a linear minimum mean square error (MMSE) equalizer. Our contribution is to derive a closed form for the mean square error (MSE) of the equalized data symbols for arbitrary correlation structure between the antennas by allowing any covariance matrix of the uplink channel. This form is powerful, because it includes not only the pilot and data transmit power levels as independent variables, and the number of receive antennas at the base station $\left(N_{r}\right)$, but can also explicitly take into account antenna spacing and the statistics of the angle of arrival (AoA), including the angular spread as a parameter. For example, the methodology allows us to study the impact of PDPR on the uplink performance for the popular spatial channel model often used to model the wireless channels in cellular systems. This formula allows us to study the impact of $N_{r}$, AoA and angular spread on the MSE and thereby on the PDPR that minimizes the MSE. To the best of our knowledge the MSE formula as well as the insights obtained in the numerical section are novel.

The system model is defined in Section II. In this section, for the sake of completeness and readability, we restate and reuse some results of [5]. The MSE for a specific channel estimate is determined in Section III, while an exact method to calculate the unconditional MSE with arbitrary channel covariance matrix is developed in Section IV. Numerical results are studied in Section V. Section VI concludes the paper.

\section{SySTEM MODEL}

We consider the uplink transmission of a SIMO single cell multi-user wireless system, in which users are scheduled on orthogonal frequency channels. It is assumed that each mobile station (MS) employs an orthogonal pilot sequence, so that no interference between pilots is present in the system. This is a 
common assumption in massive multi-user MIMO systems in which a single MS may have a single antenna [8]. Since the channel is quasi-static frequency-flat within each transmission block, it is equivalent to model the whole pilot sequence as a single symbol per resource block with power $P^{p}$, while each data symbol is transmitted with power $P$. The base station (BS) estimates the channel $\mathbf{h}$ (column vector of dimension $N_{r}$, where $N_{r}$ is the number of receive antennas at the BS) by ML channel estimators to initialize MMSE equalizers.

\section{A. Channel Estimation Model}

Each MS transmits an orthogonal pilot symbol $x_{j}$ that is received by the BS. Thus, the column vector of the received pilot signal at the BS from the $j^{\text {th }} \mathrm{MS}$ is:

$$
\mathbf{y}_{j}^{p}=\sqrt{P_{j}^{p}} \alpha_{j} \mathbf{h}_{j} x_{j}+\mathbf{n}^{p},
$$

where we assume that $\mathbf{h}_{j}$ is a circular symmetric complex normal distributed vector of r.v. with mean vector $\mathbf{0}$ and covariance matrix $\mathbf{C}_{j}$ (of size $N_{r}$ ), denoted as $\mathbf{h}_{j} \sim \mathcal{C N}\left(\mathbf{0}, \mathbf{C}_{j}\right.$ ), $\alpha_{j}$ accounts for the propagation loss, $\mathbf{n}^{p} \sim \mathcal{C N}\left(\mathbf{0}, \sigma^{2} \mathbf{I}\right)$ is the contribution from additive Gaussian noise and the pilot symbol is scaled as $\left|x_{j}\right|^{2}=1, \forall j$. Since we assume orthogonal pilot sequences, the channel estimation process can be assumed independent for each MS and we can therefore drop the index $j$. With a ML channel estimator, the BS estimates the channel based on (1) assuming

$$
\hat{\mathbf{h}}=\frac{\mathbf{y}^{p}}{\sqrt{P^{p}} \alpha x},
$$

that is:

$$
\hat{\mathbf{h}}=\mathbf{h}+\frac{\mathbf{n}^{p}}{\sqrt{P^{p}} \alpha x} ; \quad|x|^{2}=1 .
$$

It then follows that the estimated channel $\hat{\mathbf{h}}$ is distributed as follows:

$$
\hat{\mathbf{h}} \sim \mathcal{C N}(\mathbf{0}, \mathbf{R}),
$$

with $\mathbf{R} \triangleq \mathcal{E}\left[\hat{\mathbf{h}} \hat{\mathbf{h}}^{H}\right]=\mathbf{C}+\frac{\sigma^{2}}{P^{p} \alpha^{2}} \mathbf{I}$.

Further, it follows that the channel estimation error $\mathbf{w} \triangleq$ $\hat{\mathbf{h}}-\mathbf{h}$ is also normally distributed with a covariance inversely proportional to the employed pilot power:

$$
\mathbf{w} \sim \mathcal{C N}\left(\mathbf{0}, \mathbf{C}_{\mathbf{w}}\right) ; \quad \mathbf{C}_{\mathbf{w}} \triangleq \frac{\sigma^{2}}{P^{p} \alpha^{2}} \mathbf{I} .
$$

Equations (2)-(3) imply that $\mathbf{h}$ and $\hat{\mathbf{h}}$ are jointly circular symmetric complex Gaussian (multivariate normal) distributed random variables [9] [10]. Specifically, we recall from [9] that the covariance matrix of the joint PDF is composed by autocovariance matrices $\mathbf{C}_{\mathbf{h}, \mathbf{h}}, \mathbf{C}_{\hat{\mathbf{h}}, \hat{\mathbf{h}}}$ and cross covariance matrices $\mathbf{C}_{\mathbf{h}, \hat{\mathbf{h}}}, \mathbf{C}_{\hat{\mathbf{h}}, \mathbf{h}}$ as

$$
\left[\begin{array}{ll}
\mathbf{C}_{\mathbf{h}, \mathbf{h}} & \mathbf{C}_{\mathbf{h}, \hat{\mathbf{h}}} \\
\mathbf{C}_{\hat{\mathbf{h}}, \mathbf{h}} & \mathbf{C}_{\hat{\mathbf{h}}, \hat{\mathbf{h}}}
\end{array}\right]=\left[\begin{array}{ll}
\mathbf{C} & \mathbf{C} \\
\mathbf{C} & \mathbf{R}
\end{array}\right],
$$

and $\mathbf{R}=\mathbf{C}+\mathbf{C}_{\mathbf{w}}$.

\section{B. Determining the Conditional Channel Distribution}

From the joint PDF of $\mathbf{h}$ and $\hat{\mathbf{h}}$ we can compute the following conditional distributions.

Result 2.1: Given a random channel realization $\mathbf{h}$, the estimated channel $\hat{\mathbf{h}}$ conditioned to $\mathbf{h}$ can be shown to be distributed as

$$
(\hat{\mathbf{h}} \mid \mathbf{h}) \sim \mathbf{h}+\mathcal{C N}\left(\mathbf{0}, \mathbf{C}_{\mathbf{w}}\right) .
$$

Result 2.2: The distribution of the channel realization $\mathbf{h}$ conditioned to the estimate $\hat{\mathbf{h}}$ is normally distributed as follows:

$$
(\mathbf{h} \mid \hat{\mathbf{h}}) \sim \mathbf{D} \hat{\mathbf{h}}+\mathcal{C N}(\mathbf{0}, \mathbf{Q})
$$

where $\mathbf{D}=\mathbf{C R}^{-1}$ and $\mathbf{Q}=\mathbf{C}-\mathbf{C R}^{-1} \mathbf{C}$ [5].

To capture the tradeoff between the pilot and data power, we need to calculate the mean square error of the equalized data symbols. To this end, we consider an equalization model in the next subsection.

\section{Equalizer Model based on ML Channel Estimator}

The data signal received by the BS is

$$
\mathbf{y}=\alpha \sqrt{P} \mathbf{h} x+\mathbf{n},
$$

where $|x|^{2}=1$. We assume that the BS employs a naive MMSE equalizer, where the estimated channel (2) is taken as if it was the actual channel:

$$
\mathbf{G}=\alpha \sqrt{P} \hat{\mathbf{h}}^{H}\left(\alpha^{2} P \hat{\mathbf{h}} \hat{\mathbf{h}}^{H}+\sigma^{2} \mathbf{I}\right)^{-1} .
$$

Under this assumption, we recall the following two results from [5] as a first step towards determining unconditional MSE.

Result 2.3: Let $\operatorname{MSE}(\mathbf{h}, \hat{\mathbf{h}})=\mathcal{E}_{x, \mathbf{n}}\left\{|\mathbf{G y}-x|^{2}\right\}$ be the MSE for the equalized symbols, given the realizations of $\mathbf{h}$ and $\hat{\mathbf{h}}$. It is

$$
\begin{aligned}
\operatorname{MSE}(\mathbf{h}, \hat{\mathbf{h}}) & =\alpha^{2} P \mathbf{G h} \mathbf{h h}^{H} \mathbf{G}^{H}- \\
& -2 \alpha \sqrt{P} \operatorname{Re}[\mathbf{G h}]+\sigma^{2} \mathbf{G} \mathbf{G}^{H}+1 .
\end{aligned}
$$

From this, our next result follows directly.

Result 2.4: Let $\operatorname{MSE}(\hat{\mathbf{h}})=\mathcal{E}_{\mathbf{h} \mid \hat{\mathbf{h}}}\{\operatorname{MSE}(\mathbf{h}, \hat{\mathbf{h}})\}$ be the $\operatorname{MSE}$ for the equalized symbols, given the estimated channel realization $\hat{\mathbf{h}}$. It satisfies

$$
\begin{aligned}
\operatorname{MSE}(\hat{\mathbf{h}}) & =\mathbf{G}\left(\alpha^{2} P\left(\mathbf{D} \hat{\mathbf{h}} \hat{\mathbf{h}}^{H} \mathbf{D}^{H}+\mathbf{Q}\right)+\sigma^{2} \mathbf{I}\right) \mathbf{G}^{H}- \\
& -2 \alpha \sqrt{P} \operatorname{Re}\{\mathbf{G D} \hat{\mathbf{h}}\}+1
\end{aligned}
$$

\section{Determining the MSE As a FunCtion of $\hat{\mathbf{h}}$}

Based on the conditional MSE expression of the preceding section, we are now interested in deriving the unconditional expectation of the MSE. To this end, the following two lemmas turn out to be useful.

Lemma 1: Given a channel estimate instance $\hat{\mathbf{h}}$, the MMSE weighting matrix $\mathbf{G}$, as a function of the number of receive antennas at the base station $\left(N_{r}\right)$ can be expressed as follows

$$
\mathbf{G}=\frac{\alpha \sqrt{P}}{\|\hat{\mathbf{h}}\|^{2} \alpha^{2} P+\sigma^{2}} \hat{\mathbf{h}}^{H},
$$


where $\|\hat{\mathbf{h}}\|^{2}=\hat{\mathbf{h}}^{H} \hat{\mathbf{h}}=\sum_{i=1}^{N_{r}}\left|\hat{h}_{i}\right|^{2}$.

The proof is in the Appendix.

Using $z=\frac{\alpha \sqrt{P}}{\|\hat{\mathbf{h}}\|^{2} \alpha^{2} P+\sigma^{2}}$ and this simple expression of $\mathbf{G}$ we can express the conditional expectation of the MSE of the MMSE equalized data symbols as a function of the channel covariance, $\mathbf{C}$.

Lemma 2:

$$
\begin{aligned}
\operatorname{MSE}(\hat{\mathbf{h}}) & =z \hat{\mathbf{h}}^{H}\left(\alpha^{2} P\left(\mathbf{D} \hat{\mathbf{h}} \hat{\mathbf{h}}^{H} \mathbf{D}^{H}+\mathbf{Q}\right)+\sigma^{2} \mathbf{I}\right) z \hat{\mathbf{h}}- \\
& -2 \alpha \sqrt{P} \operatorname{Re}\left\{z \hat{\mathbf{h}}{ }^{H} \mathbf{D} \hat{\mathbf{h}}\right\}+1= \\
& =z^{2} \alpha^{2} P \hat{\mathbf{h}}^{H} \mathbf{D} \hat{\mathbf{h}} \hat{\mathbf{h}}^{H} \mathbf{D}^{H} \hat{\mathbf{h}}+z^{2} \alpha^{2} P \hat{\mathbf{h}}^{H} \mathbf{Q} \hat{\mathbf{h}}+ \\
& +z^{2} \sigma^{2} \hat{\mathbf{h}}^{H} \hat{\mathbf{h}}-2 z \alpha \sqrt{P} \operatorname{Re}\left\{\hat{\mathbf{h}}^{H} \mathbf{D} \hat{\mathbf{h}}\right\}+1 .
\end{aligned}
$$

where $\mathbf{D}=\mathbf{C}\left(\mathbf{C}+\frac{\sigma^{2}}{P^{p} \alpha^{2}} \mathbf{I}\right)^{-1}, \mathbf{Q}=\mathbf{C}-\mathbf{C}\left(\mathbf{C}+\frac{\sigma^{2}}{P^{p} \alpha^{2}} \mathbf{I}\right)^{-1} \mathbf{C}$ and $z=\frac{\alpha \sqrt{P}}{\|\hat{\mathbf{h}}\|^{2} \alpha^{2} P+\sigma^{2}}$ is a function of $\|\hat{\mathbf{h}}\|^{2}$.

Proof: Substituting (10) into (9) gives the lemma.

\section{Approximation Method Using the $\Gamma$ Function}

In this section, our goal is to compute $\mathcal{E}_{\hat{\mathbf{h}}}\{\operatorname{MSE}(\hat{\mathbf{h}})\}$ based on Lemma 2 for arbitrary covariance matrices $\mathbf{C}$. To this end, a lemma that allows us to calculate $z$ and the singular value decomposition of $\mathbf{C}$ are instrumental.

\section{A. Computing $z$}

Computing $z$ in Lemma 2 is essential in calculating the unconditional MSE. This can be done using the following lemma.

Lemma 3:

$$
z=\frac{1}{\alpha \sqrt{P}} \cdot \int_{x=0}^{\infty} e^{-x\left(\hat{\mathbf{h}}^{H} \hat{\mathbf{h}}+\sigma^{2} /\left(\alpha^{2} P\right)\right)} d x
$$

and

$$
z^{2}=\frac{1}{\alpha^{2} P} \cdot \int_{x=0}^{\infty} x e^{-x\left(\hat{\mathbf{h}}^{H} \hat{\mathbf{h}}+\sigma^{2} /\left(\alpha^{2} P\right)\right)} d x .
$$

The proof is in the Appendix.

\section{B. Singular Value Decomposition (SVD) of $\mathbf{C}$}

To take the expectation with respect to $\hat{\mathbf{h}}$ of the terms of $\operatorname{MSE}(\hat{\mathbf{h}})$ as in Lemma 2, we need the following decomposition. $\hat{\mathbf{h}}$ is $\mathcal{C N}(\mathbf{0}, \mathbf{R})$ distributed with $\mathbf{R}=\mathbf{C}+\frac{\sigma^{2}}{P^{p} \alpha^{2}} \mathbf{I}$ and we recall from Result 2.2 that $\mathbf{D}=\mathbf{C R}^{-1}$. Let $\mathbf{C}=\mathbf{\Theta}^{H} \mathbf{S}_{\mathbf{C}} \boldsymbol{\Theta}$ be the singular value decomposition of $\mathbf{C}$. Then $\mathbf{R}=\boldsymbol{\Theta}^{H} \mathbf{S}_{\mathbf{R}} \boldsymbol{\Theta}$ and $\mathbf{D}=\boldsymbol{\Theta}^{H} \mathbf{S}_{\mathbf{D}} \boldsymbol{\Theta}$ with $\mathbf{S}_{\mathbf{R}}=\mathbf{S}_{\mathbf{C}}+\frac{\sigma^{2}}{P^{p} \alpha^{2}} \mathbf{I}$ and $\mathbf{S}_{\mathbf{D}}=\mathbf{S}_{\mathbf{C}} \mathbf{S}_{\mathbf{R}}{ }^{-1}$, where matrices $\mathbf{S}$. are real non-negative diagonal matrices. Specifically, we will refer to the diagonal elements of $\mathbf{D}$ and $\mathbf{R}$ using the notations $d_{k}=\mathbf{S}_{\mathbf{D} k k}$ and $r_{k}=\mathbf{S}_{\mathbf{R} k k}$ respectively. Let $\mathbf{v}=\boldsymbol{\Theta} \hat{\mathbf{h}}$, then $\mathbf{v}$ is a random vector with distribution $\mathcal{C N}\left(\mathbf{0}, \mathbf{S}_{\mathbf{R}}\right)$, since

$$
\begin{gathered}
\mathcal{E}\left(\mathbf{v} \mathbf{v}^{H}\right)=\mathcal{E}\left(\boldsymbol{\Theta} \hat{\mathbf{h}} \hat{\mathbf{h}}^{H} \boldsymbol{\Theta}^{H}\right)=\boldsymbol{\Theta} \mathcal{E}\left(\hat{\mathbf{h}} \hat{\mathbf{h}}^{H}\right) \boldsymbol{\Theta}^{H}= \\
=\boldsymbol{\Theta} \mathbf{R} \Theta^{H}=\boldsymbol{\Theta} \Theta^{H} \mathbf{S}_{\mathbf{R}} \boldsymbol{\Theta} \Theta^{H}=\mathbf{S}_{\mathbf{R}} .
\end{gathered}
$$

That is, the elements of $\mathbf{v}$ are independent, but they have different variances.
C. Terms of $\mathcal{E}\{M S E\}=\mathcal{E}_{\hat{\mathbf{h}}}\{\operatorname{MSE}(\hat{\mathbf{h}})\}$

To compute $\mathcal{E}_{\hat{\mathbf{h}}}\{\operatorname{MSE}(\hat{\mathbf{h}})\}$ based on Lemma 2, we need to calculate the following terms $\left(T_{1}, T_{2}\right.$ and $\left.T_{3}\right)$ :

$$
\begin{aligned}
\mathcal{E}_{\hat{\mathbf{h}}}\{\operatorname{MSE}(\hat{\mathbf{h}})\}= & \underbrace{\alpha^{2} P \mathcal{E}_{\hat{\mathbf{h}}}\left\{z^{2} \hat{\mathbf{h}}^{H} \mathbf{D} \hat{\mathbf{h}} \hat{\mathbf{h}}^{H} \mathbf{D}^{H} \hat{\mathbf{h}}\right\}}_{T_{1}}+ \\
& +\underbrace{\mathcal{E}_{\hat{\mathbf{h}}\left\{z^{2} \hat{\mathbf{h}}^{H}\left(\alpha^{2} P \mathbf{Q}+\sigma^{2} \mathbf{I}\right) \hat{\mathbf{h}}\right\}}}_{T_{2}}+ \\
& +\underbrace{\left.2 \alpha \sqrt{P} \mathcal{E}_{\hat{\mathbf{h}}\left\{z \operatorname{Re}\left\{\hat{\mathbf{h}}^{H} \mathbf{D} \hat{\mathbf{h}}\right\}\right\}}\right\}}_{T_{3}} .
\end{aligned}
$$

With Lemmas 1, 2 and 3 in our hands, we can state our main result on calculating the terms $T_{1}, T_{2}$ and $T_{3}$, whereas for the proofs we refer to the Appendix.

Theorem 1: The mean square error (MSE) of the uplink received data with arbitrary correlation matrix $\mathbf{C}$ of the uplink channel can be calculated as in the sum of the terms $T_{1}, T_{2}$ and $T_{3}$, where

$$
\begin{aligned}
T_{1}= & \sum_{k} \sum_{\ell, \ell \neq k} d_{k} d_{\ell} \\
& \cdot \int_{x=0}^{\infty} x e^{-x \sigma^{2} /\left(\alpha^{2} P\right)} \frac{1}{x+r_{k}} \frac{1}{x+r_{\ell}} \prod_{i} \frac{r_{i}}{x+r_{i}} d x+ \\
& +\sum_{k} d_{k}^{2} \int_{x=0}^{\infty} x e^{-x \sigma^{2} /\left(\alpha^{2} P\right)} \frac{2}{\left(x+r_{k}\right)^{2}} \prod_{i} \frac{r_{i}}{x+r_{i}} d x \\
T_{2}= & \frac{1}{\alpha^{2} P} \sum_{k} \mathbf{S}_{\mathbf{M} k k} \int_{x=0}^{\infty} x e^{-x \frac{\sigma^{2}}{\alpha^{2} P}} \frac{1}{x+r_{k}} \prod_{i} \frac{r_{i}}{x+r_{i}} d x
\end{aligned}
$$

and

$$
T_{3}=2 \sum_{k} d_{k} \int_{x=0}^{\infty} e^{-x \frac{\sigma^{2}}{\alpha^{2} P}} \frac{1}{x+r_{k}} \prod_{i} \frac{r_{i}}{x+r_{i}} d x,
$$

where $\mathbf{S}_{\mathbf{M}}=\alpha^{2} P \mathbf{S}_{\mathbf{Q}}+\sigma^{2} \mathbf{I}$ is a diagonal matrix with diagonal elements $\mathbf{S}_{\mathbf{M} k k}=\alpha^{2} P \mathbf{S}_{\mathbf{Q}_{k k}}+\sigma^{2}$.

\section{Numerical Results}

\section{A. Channel Model and Covariance Matrix}

TABLE I

SYSTEM PARAMETERS

\begin{tabular}{l|l}
\hline \hline Parameter & Value \\
\hline \hline Number of antennas & $N_{r}=2,4,8,10,20$ \\
\hline Path Loss & $\alpha=50,55,60 \mathrm{~dB}$ \\
\hline Power budget & $P^{p}+P=250 \mathrm{~mW}$ \\
\hline Antenna apacing & $D / \lambda=0.15 \ldots 1.5$ \\
\hline & $\bar{\theta}=70^{\circ}$ \\
\hline Mean Angle of Arrival (AoA) & $2 \cdot \theta_{\Delta}=5 \ldots 45^{\circ}$ \\
\hline Angular spread &
\end{tabular}

In this section we consider a single cell system, in which MSs use orthogonal pilots to facilitate the estimation of the uplink channel by the BS. Recall from Section II-A that the channel estimation process is independent for each MS and we can therefore focus on a single user. The covariance matrix $\mathbf{C}$ 
of the channel $\mathbf{h}$ as the function of the antenna spacing, mean angle of arrival and angular spread is modeled as by the well known spatial channel model, see [11]:

$$
\mathbf{C}(m, n)=\frac{\alpha}{2 \theta_{\Delta}} \int_{-\theta_{\Delta}}^{\theta_{\Delta}} e^{j \cdot 2 \pi \cdot \frac{D}{\lambda}(n-m) \cos (\bar{\theta}+x)} d x,
$$

where the system parameters are given in Table I. The covariance matrix $\mathbf{C}$ becomes practically diagonal as the antenna spacing and the angular spread grows beyond $D \lambda>1$ and $\theta_{\Delta}>30^{\circ}$. In contrast, with critically spaced antennas $D \lambda=0.5$ and $\theta_{\Delta}<10^{\circ}$, the antenna correlation in terms of the off-diagonal elements of $\mathbf{C}$ can be considered strong.

\section{B. Numerical Results}

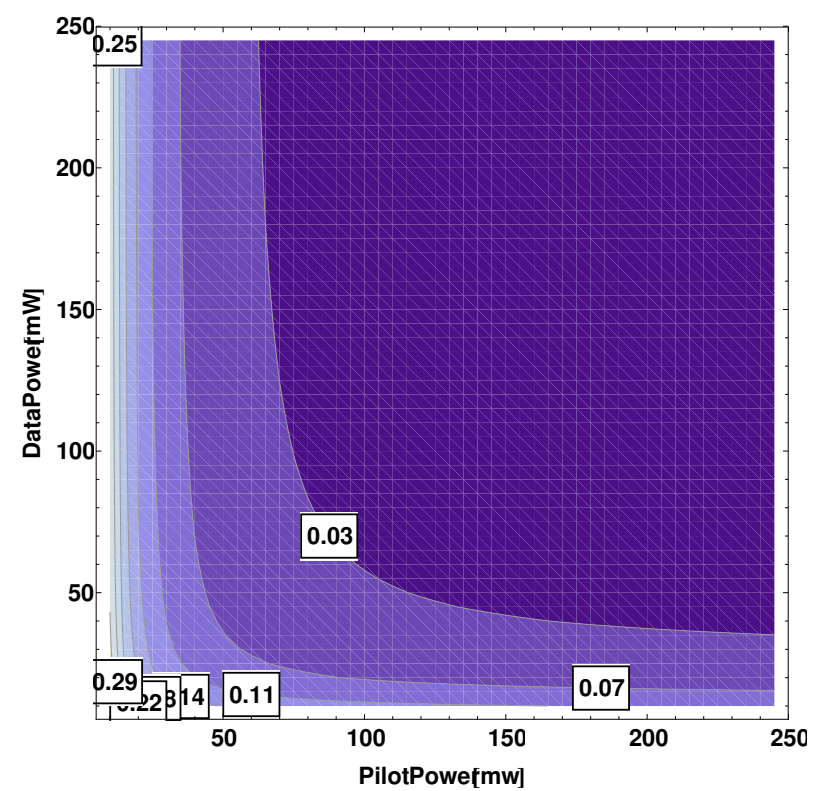

Fig. 1. Contour plot of the MSE achieved by specific pilot and data power settings of a SIMO system with $N_{r}=20$ uncorrelated receiver antennas at a fix path loss position of $50 \mathrm{~dB}$. For example the MSE value of 0.03 can be reached by setting the pilot power to $P^{p}=100 \mathrm{~mW}$ and the data power $P=60 \mathrm{~mW}$, or by $P^{p}=200 \mathrm{~mW}$ and $P=30 \mathrm{~mW}$. We can see that with a total power budget of $P^{p}+P=250 \mathrm{~mW}$, and with proper pilot-data balancing, a minimum MSE that is clearly less than 0.03 can be reached.

Figures 1 and 2 compare the MSE that can be achieved by a particular setting of the pilot and data power levels in the case of uncorrelated and highly correlated antennas (for the case with $N_{r}=20$ receive antennas). The impact of high antenna correlation is that in order to reach the same MSE, a higher power level for both the pilot and data transmission must be used.

Figure 3 shows the percentage of the total power budget that minimizes the MSE as a function of the path loss between the $\mathrm{MS}$ and the BS when the number of BS antennas is varied as $N_{r}=2,4,8,10,20$. Here we can clearly see the at a given position (path loss), the pilot-to-data power ratio (PDPR) increases with the number of antennas. The intuition behind this behavior is that at a larger number of antennas, the data transmit power can be lower than with a few antennas and a higher pilot power level (a higher PDPR) can be afforded by

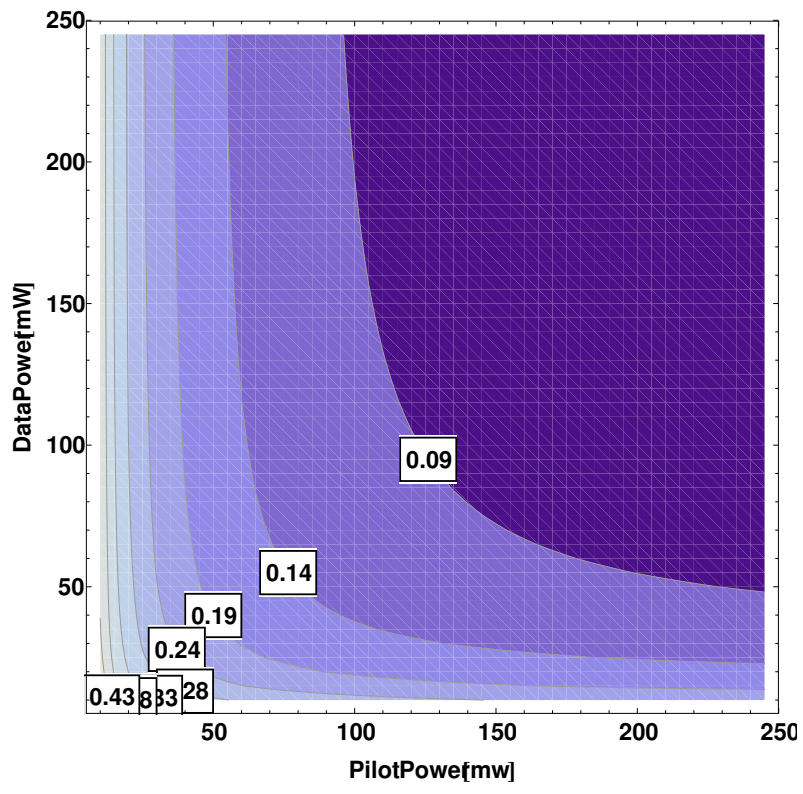

Fig. 2. Contour plot of the MSE achieved by specific pilot and data power settings of a SIMO system with $N_{r}=20$ correlated receiver antennas at a fix path loss position of $50 \mathrm{~dB}$. Compared with Figure 1, we can see that with similar sum power budget, the MSE value that can be reached is somewhat higher. For example, with a power budget of $250 \mathrm{~mW}$, an MSE value that is less than 0.09 can be realized $\left(P^{p}=150\right.$ and $\left.P=100 \mathrm{~mW}\right)$.

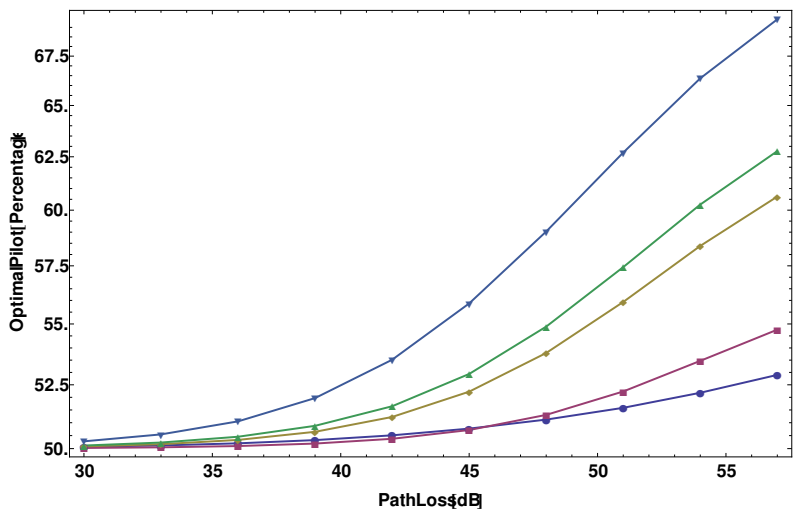

Fig. 3. The percentage of the total power budget that minimizes the MSE as a function of the path loss for $N_{r}=2,4,8,10,20$ antennas. $\left(N_{r}=20\right.$ corresponds to the uppermost curve.) At a given path loss, the percentage of the power budget that minimizes the MSE increases with the number of antennas.0

the MS. Interestingly, the PDPR that minimizes the MSE, in this example (and also in other examples not reported here) is virtually the same for the uncorrelated and correlated antenna cases.

The value of the minimum MSE, assuming the optimal PDPR setting is shown by Figure 4 and Figure 5 for the case of uncorrelated and correlated antennas respectively. By examining Figures 4-5, we can see that the impact of (high) antenna correlation is significant. For example with $N_{r}=20$ receive antennas, at $50 \mathrm{~dB}$ path loss, the minimum MSE (that is with the optimal PDPR) is around 0.004, whereas with correlated antennas, the minimum MSE is around 0.010.

Next, we examine the impact of antenna spacing and com- 


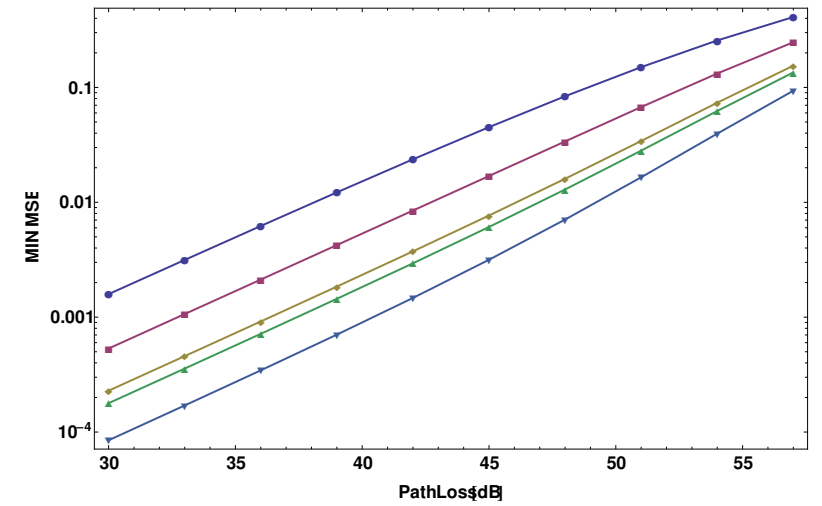

Fig. 4. The minimum MSE that can be reached by setting the percentage of the pilot power according to Figure 3 when the antennas are uncorrelated. ( $N_{r}=20$ corresponds to the lowermost curve.) For example, at path loss 50 $\mathrm{dB}$ an order of magnitude lower MSE can be reached with $N_{r}=20$ antennas than with $N_{r}=2$.

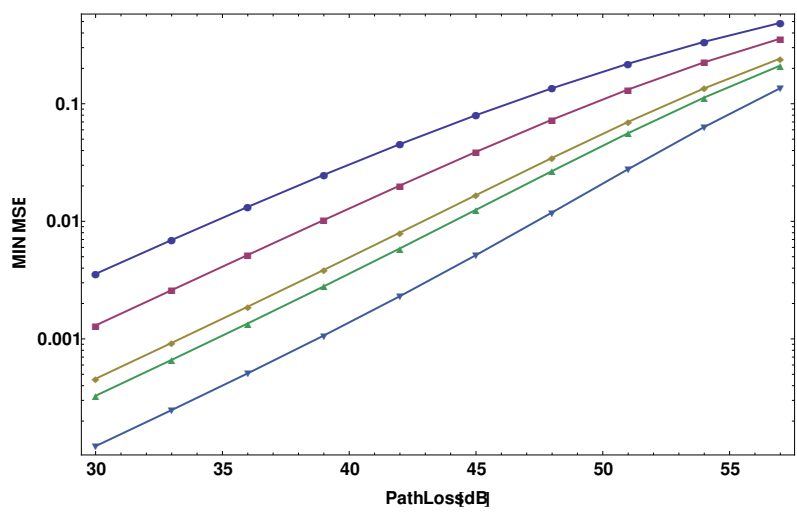

Fig. 5. The minimum MSE that can be reached by setting the percentage of the pilot power according to Figure 3 when the antennas are correlated. ( $N_{r}=20$ corresponds to the lowermost curve.) Compared with Figure 4, we can see that the MSE values get somewhat higher.

pare that with the impact of PDPR setting on the MSE in Figure 6. In this figure, the uppermost and the middle curves correspond to suboptimal PDPR settings. The insight provided by this figure is important because it suggests that with correct PDPR setting, the negative impact of antenna corrleation can be compensated. In fact, the correct setting of the PDPR is more important than decreasing the antenna correlation by, for example, increasing the antenna spacing between antenna elements.

Finally, Figure 7 shows the MSE as a function of the angular spread (AS) and the pilot power when the total power budget is $300 \mathrm{~mW}$. Similarly to Figure 6, we can observe that although an increasing AS have a clear positive effect on the MSE, setting the right pilot power level can to a large extent mitigate the impact of a small AS on the MSE.

\section{CONCLUSIONS}

In this paper we developed a model of a single cell system, in which MSs use orthogonal pilots to facilitate uplink channel estimation by the BS. We developed a methodology to calculate the MSE of the uplink equalized data symbols and derived a closed form for the MSE as a function of not only the number

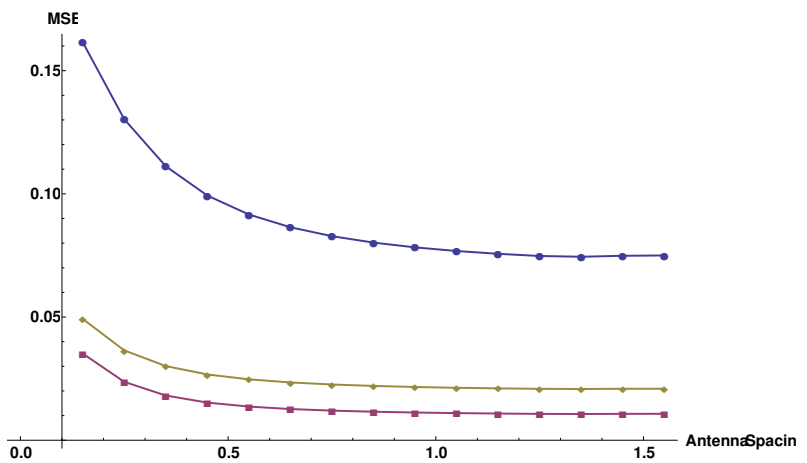

Fig. 6. The impact of antenna spacing $\left(N_{r}=20\right)$ on the MSE at path loss of $50 \mathrm{~dB}$. The three curves correspond to the optimal pilot power setting (lowest curve), too high pilot power (middle) and too low pilot power levels (uppermost).

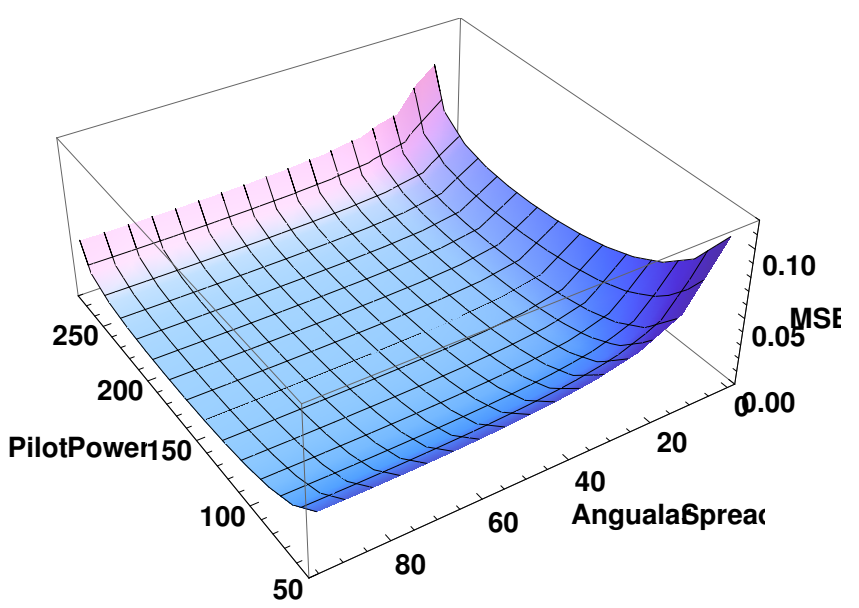

Fig. 7. The MSE as a function of the angular spread and the pilot power. A larger angular spread implies a richer SIMO channel and thereby a lower MSE value for all values of the pilot power.

of antennas, the pilot power and the data transmit power, but also the path loss and other parameters that determine the covariance matrix of the fast fading channel between the MS and the BS. With this methodology, through numerical examples, we found that although the impact of antenna correlation on the MSE performance can be significant, this impact can be compensated by setting the correct pilot-todata power ratio (PDPR) in case of a total power budget. Furthermore, we found that as the number of antennas grows, a higher ratio of the power budget should be spent on pilots, virtually independently of the antenna correlation. This can be seen in line with the findings of massive MIMO systems that suggest that the data transmit power at the MS can be significantly lower as the number of antennas at the BS grows large.

\section{APPENDIX}

\section{A. Proof or Lemma 1}

According to the matrix inversion lemma for matrices $\mathbf{A}$, $\mathbf{B}, \mathbf{C}, \mathbf{D}$ of size $n \times n, n \times m, m \times m, m \times n$, respectively, 
we have

$$
\begin{aligned}
& (\mathbf{A}+\mathbf{B C D})^{-1}= \\
& \mathbf{A}^{-1}-\mathbf{A}^{-1} \mathbf{B}\left(\mathbf{D A}{ }^{-1} \mathbf{B}+\mathbf{C}^{-1}\right)^{-1} \mathbf{D A} \mathbf{A}^{-1} .
\end{aligned}
$$

Substituting $\mathbf{A}=\sigma^{2} \mathbf{I}, \mathbf{B}=\alpha \sqrt{P} \hat{\mathbf{h}}, \mathbf{C}=1, \mathbf{D}=\alpha \sqrt{P} \hat{\mathbf{h}}^{H}$ we have

$$
\begin{aligned}
& \left(\sigma^{2} \mathbf{I}+\alpha^{2} P \hat{\mathbf{h}} \hat{\mathbf{h}}^{H}\right)^{-1}=\frac{1}{\sigma^{2}} \mathbf{I}-\frac{1}{\sigma^{2}} \mathbf{I} \alpha \sqrt{P} \hat{\mathbf{h}} . \\
& \cdot\left(\alpha \sqrt{P} \hat{\mathbf{h}}^{H} \frac{1}{\sigma^{2}} \mathbf{I} \alpha \sqrt{P} \hat{\mathbf{h}}+1\right)^{-1} \alpha \sqrt{P} \hat{\mathbf{h}}^{H} \frac{1}{\sigma^{2}} \mathbf{I}= \\
& =\frac{1}{\sigma^{2}} \mathbf{I}-\frac{\frac{\alpha^{2} P}{\sigma^{4}} \hat{\mathbf{h}} \hat{\mathbf{h}}^{H}}{\frac{\alpha^{2} P}{\sigma^{2}} \hat{\mathbf{h}} \hat{\mathbf{h}}^{H}+1},
\end{aligned}
$$

where $\hat{\mathbf{h}}^{H} \hat{\mathbf{h}}=\|\hat{\mathbf{h}}\|^{2}$. Finally,

$$
\begin{aligned}
\mathbf{G} & =\alpha \sqrt{P} \hat{\mathbf{h}}^{H}\left(\alpha^{2} P \hat{\mathbf{h}} \hat{\mathbf{h}}^{H}+\sigma^{2} \mathbf{I}\right)^{-1} \\
& =\frac{\alpha \sqrt{P}}{\sigma^{2}} \hat{\mathbf{h}}^{H}-\frac{\alpha \sqrt{P} \frac{\alpha^{2} P}{\sigma^{4}} \hat{\mathbf{h}}^{H} \hat{\mathbf{h}}}{\frac{\alpha^{2} P}{\sigma^{2}}\|\hat{\mathbf{h}}\|^{2}+1} \hat{\mathbf{h}}^{H} \\
& =\frac{\alpha \sqrt{P}}{\sigma^{2}} \hat{\mathbf{h}}^{H}\left(1-\frac{\alpha^{2} P\|\hat{\mathbf{h}}\|^{2}}{\alpha^{2} P\|\hat{\mathbf{h}}\|^{2}+\sigma^{2}}\right) \\
& =\frac{\alpha \sqrt{P}}{\sigma^{2}} \hat{\mathbf{h}}^{H}\left(\frac{\sigma^{2}}{\alpha^{2} P\|\hat{\mathbf{h}}\|^{2}+\sigma^{2}}\right) \\
& =\frac{\alpha \sqrt{P}}{\alpha^{2} P\|\hat{\mathbf{h}}\|^{2}+\sigma^{2}} \hat{\mathbf{h}}^{H} .
\end{aligned}
$$

\section{B. Proof of Lemma 3}

First, we notice that according to equation 3.326 (2) of [12]:

$$
\int_{x=0}^{\infty} x^{m} e^{-y x^{n}} d x=\frac{\Gamma(\gamma)}{n y^{\gamma}} ; \quad \gamma=\frac{m+1}{n},
$$

which specifically for $n=1$ means:

$$
\frac{1}{y^{\gamma}}=\int_{x=0}^{\infty} \frac{x^{\gamma-1}}{\Gamma(\gamma)} e^{-x y} d x .
$$

that is, for $\gamma=1$ and $y=\|\hat{\mathbf{h}}\|^{2}+\sigma^{2} /\left(\alpha^{2} P\right)$ :

$$
\begin{aligned}
z & =\frac{\alpha \sqrt{P}}{\|\hat{\mathbf{h}}\|^{2} \alpha^{2} P+\sigma^{2}}= \\
& =\frac{1}{\alpha \sqrt{P}} \frac{1}{\|\hat{\mathbf{h}}\|^{2}+\sigma^{2} /\left(\alpha^{2} P\right)}= \\
& =\frac{1}{\alpha \sqrt{P} \underbrace{\Gamma(1)}_{1}} \cdot \int_{x=0}^{\infty} e^{-x\left(\hat{\mathbf{h}}^{H} \hat{\mathbf{h}}+\sigma^{2} /\left(\alpha^{2} P\right)\right)} d x
\end{aligned}
$$

and, for $\gamma=2$ :

$$
\begin{aligned}
z^{2} & =\frac{\alpha^{2} P}{\left(\|\hat{\mathbf{h}}\|^{2} \alpha^{2} P+\sigma^{2}\right)^{2}}= \\
& =\frac{1}{\alpha^{2} P \underbrace{\Gamma(2)}_{1}} \cdot \int_{x=0}^{\infty} x e^{-x\left(\hat{\mathbf{h}}^{H} \hat{\mathbf{h}}+\sigma^{2} /\left(\alpha^{2} P\right)\right)} d x .
\end{aligned}
$$

\section{Term $T_{1}$}

Using the SVD based transformation as in Section IV-B $\mathbf{v}=\boldsymbol{\Theta} \hat{\mathbf{h}}$ and Lemma 3, we can write:

$$
T_{1}=\alpha^{2} P \mathcal{E}_{\hat{\mathbf{h}}}\left\{z^{2} \hat{\mathbf{h}}^{H} \mathbf{D} \hat{\mathbf{h}} \hat{\mathbf{h}}^{H} \mathbf{D}^{H} \hat{\mathbf{h}}\right\}=
$$

$$
\begin{aligned}
&= \mathcal{E}_{\hat{\mathbf{h}}}\left\{\int_{x=0}^{\infty} x e^{-x\left(\hat{\mathbf{h}}^{H} \hat{\mathbf{h}}+\sigma^{2} /\left(\alpha^{2} P\right)\right)} d x \hat{\mathbf{h}}^{H} \mathbf{D} \hat{\mathbf{h}} \hat{\mathbf{h}}^{H} \mathbf{D}^{H} \hat{\mathbf{h}}\right\}= \\
&= \mathcal{E}_{\mathbf{v}}\left\{\int_{x=0}^{\infty} x e^{-x\left(\mathbf{v}^{H} \mathbf{v}+\sigma^{2} /\left(\alpha^{2} P\right)\right)} d x \mathbf{v}^{H} \mathbf{S}_{\mathbf{D}} \mathbf{v} \mathbf{v}^{H} \mathbf{S}_{\mathbf{D}}{ }^{H} \mathbf{v}\right\}= \\
&= \mathcal{E}_{\mathbf{v}}\left\{\int_{x=0}^{\infty} x e^{-x\left(\sum_{i} v_{i}^{H} v_{i}+\sigma^{2} /\left(\alpha^{2} P\right)\right)} d x \cdot\right. \\
&=\int_{x=0}^{\infty} x e^{-x \sigma^{2} /\left(\alpha^{2} P\right)} \cdot \mathcal{E}_{\mathbf{v}}\left\{\prod_{i} e^{-x\left(v_{i}^{H} v_{i}\right)} \cdot\left(\sum_{\ell} v_{k}^{H} \mathbf{S}_{\mathbf{D} k k} v_{k}\right)\left(\mathbf{S}_{\mathbf{D} \ell \ell} v_{\ell}\right)\right\}= \\
&\left.\cdot\left(\sum_{k} v_{k}^{H} \mathbf{S}_{\mathbf{D} k k} v_{k}\right)\left(\sum_{\ell} v_{\ell}^{H} \mathbf{S}_{\mathbf{D} \ell \ell} v_{\ell}\right)\right\} d x .
\end{aligned}
$$

The expectation in (11) can be computed as follows:

$$
\begin{aligned}
& \mathcal{E}_{\mathbf{v}}\left\{\prod_{i} e^{-x\left|v_{i}\right|^{2}} d x\left(\sum_{k} \mathbf{S}_{\mathbf{D} k k}\left|v_{k}\right|^{2}\right)\left(\sum_{\ell} \mathbf{S}_{\mathbf{D} \ell \ell}\left|v_{\ell}\right|^{2}\right)\right\}= \\
& =\sum_{k} \sum_{\ell, \ell \neq k} \mathbf{S}_{\mathbf{D} k k} \mathbf{S}_{\mathbf{D} \ell \ell} .
\end{aligned}
$$

$$
\cdot \mathcal{E}_{\mathbf{v}}\{e^{-x\left|v_{k}\right|^{2}}\left|v_{k}\right|^{2} e^{-x\left|v_{\ell}\right|^{2}}\left|v_{\ell}\right|^{2} \underbrace{\prod_{i, i \neq k, i \neq \ell} e^{-x\left|v_{i}\right|^{2}}}_{n-2 \text { terms }}\}+
$$$$
+\sum_{k} \mathbf{S}_{\mathbf{D} k k}^{2} \mathcal{E}_{\mathbf{v}}\{e^{-x\left|v_{k}\right|^{2}}\left|v_{k}\right|^{4} \underbrace{n-1 \text { terms }}_{\underbrace{\prod_{i, i \neq k} e^{-x\left|v_{i}\right|^{2}}}}\}=
$$

$$
\begin{aligned}
& =\sum_{k} \sum_{\ell, \ell \neq k} \mathbf{S}_{\mathbf{D} k k} \mathbf{S}_{\mathbf{D} \ell \ell} \mathcal{E}_{v_{k}}\left\{\left|v_{k}\right|^{2} e^{-x\left|v_{k}\right|^{2}}\right\} \\
& \quad \cdot \mathcal{E}_{v_{\ell}}\left\{\left|v_{\ell}\right|^{2} e^{-x\left|v_{\ell}\right|^{2}}\right\} \prod_{i, i \neq k, i \neq \ell} \mathcal{E}_{v_{i}}\left\{e^{-x\left|v_{i}\right|^{2}}\right\}+ \\
& +\sum_{k} \mathbf{S}_{\mathbf{D} k k}^{2} \mathcal{E}_{v_{k}}\left\{\left|v_{k}\right|^{4} e^{-x\left|v_{k}\right|^{2}}\right\} \prod_{i, i \neq k} \mathcal{E}_{v_{i}}\left\{e^{-x\left|v_{i}\right|^{2}}\right\},
\end{aligned}
$$

where $y=\left|v_{i}\right|^{2}$ is exponentially distributed with parameter $r_{i}=\mathbf{S}_{\mathbf{R} i i}$. Consequently:

$$
\begin{gathered}
\mathcal{E}_{v_{i}}\left\{e^{-x\left|v_{i}\right|^{2}}\right\}=\int_{y=0}^{\infty} r_{i} e^{-r_{i} y} e^{-x y} d y=\frac{r_{i}}{x+r_{i}} \\
\mathcal{E}_{v_{i}}\left\{\left|v_{i}\right|^{2} e^{-x\left|v_{i}\right|^{2}}\right\}=\int_{y=0}^{\infty} r_{i} e^{-r_{i} y} y e^{-x y} d y=\frac{r_{i}}{\left(x+r_{i}\right)^{2}}
\end{gathered}
$$




$$
\mathcal{E}_{v_{i}}\left\{\left|v_{i}\right|^{4} e^{-x\left|v_{i}\right|^{2}}\right\}=\int_{y=0}^{\infty} r_{i} e^{-r_{i} y} y^{2} e^{-x y} d y=\frac{2 r_{i}}{\left(x+r_{i}\right)^{3}} .
$$

Substituting all of these into the expectation and using $d_{i}=$ $\mathbf{S}_{\mathbf{D}_{i i}}$, we have:

$$
\begin{aligned}
& \sum_{k} \sum_{\ell, \ell \neq k} d_{k} d_{\ell} \mathcal{E}_{v_{k}}\left\{\left|v_{k}\right|^{2} e^{-x\left|v_{k}\right|^{2}}\right\} \mathcal{E}_{v_{\ell}}\left\{\left|v_{\ell}\right|^{2} e^{-x\left|v_{\ell}\right|^{2}}\right\} \\
& \quad \cdot \prod_{i, i \neq k, i \neq \ell} \mathcal{E}_{v_{i}}\left\{e^{-x\left|v_{i}\right|^{2}}\right\}+ \\
& \quad+\sum_{k} d_{k}^{2} \mathcal{E}_{v_{k}}\left\{\left|v_{k}\right|^{4} e^{-x\left|v_{k}\right|^{2}}\right\} \prod_{i, i \neq k} \mathcal{E}_{v_{i}}\left\{e^{-x\left|v_{i}\right|^{2}}\right\}= \\
& =\sum_{k} \sum_{\ell, \ell \neq k} d_{k} d_{\ell} \frac{r_{k}}{\left(x+r_{k}\right)^{2}} \frac{r_{\ell}}{\left(x+r_{\ell}\right)^{2}} \prod_{i, i \neq k, i \neq \ell} \frac{r_{i}}{x+r_{i}}+ \\
& \quad+\sum_{k} d_{k}^{2} \frac{2 r_{k}}{\left(x+r_{k}\right)^{3}} \prod_{i, i \neq k} \frac{r_{i}}{x+r_{i}} .
\end{aligned}
$$

Multiplying and dividing with the missing terms of the products, the expectation in (11) can be written as:

$$
\begin{aligned}
& \sum_{k} \sum_{\ell, \ell \neq k} d_{k} d_{\ell} \frac{1}{x+r_{k}} \frac{1}{x+r_{\ell}} \prod_{i} \frac{r_{i}}{x+r_{i}} \\
& +\sum_{k} d_{k}^{2} \frac{2}{\left(x+r_{k}\right)^{2}} \prod_{i} \frac{r_{i}}{x+r_{i}} .
\end{aligned}
$$

Substituting this expression for the expectation, we finally get for $T 1$ :

$$
\begin{gathered}
T 1=\int_{x=0}^{\infty} x e^{-x \sigma^{2} /\left(\alpha^{2} P\right)} \cdot \mathcal{E}_{\mathbf{v}}\left\{\prod_{i} e^{-x\left(v_{i}^{H} v_{i}\right)} .\right. \\
\left.\cdot\left(\sum_{k} v_{k}^{H} \mathbf{S}_{\mathbf{D} k k} v_{k}\right)\left(\sum_{\ell} v_{\ell}^{H} \mathbf{S}_{\mathbf{D} \ell \ell} v_{\ell}\right)\right\} d x= \\
=\sum_{k} \sum_{\ell, \ell \neq k} d_{k} d_{\ell} \int_{x=0}^{\infty} x e^{-x \sigma^{2} /\left(\alpha^{2} P\right)} \frac{r_{k}}{\left(x+r_{k}\right)^{2}} \frac{r_{\ell}}{\left(x+r_{\ell}\right)^{2}} . \\
+\sum_{k} \prod_{i, i \neq k, i \neq \ell}^{2} \frac{r_{i}}{x+r_{i}} d x+ \\
=\sum_{x=0}^{\infty} x e^{-x \sigma^{2} /\left(\alpha^{2} P\right)} \frac{2 r_{k}}{\left(x+r_{k}\right)^{3}} \prod_{i, i \neq k} \frac{r_{i}}{x+r_{i}} d x= \\
\quad \sum_{x=0}^{\infty} x e_{k}^{-x \sigma^{2} /\left(\alpha^{2} P\right)} \frac{1}{x+r_{k}} \frac{1}{x+r_{\ell}} \prod_{i} \frac{r_{i}}{x+r_{i}} d x+ \\
+\sum_{k} d_{k}^{2} \int_{x=0}^{\infty} x e^{-x \sigma^{2} /\left(\alpha^{2} P\right)} \frac{2}{\left(x+r_{k}\right)^{2}} \prod_{i} \frac{r_{i}}{x+r_{i}} d x .
\end{gathered}
$$

All of these integrals can be computed efficiently, but they do not have nice closed forms.

\section{Term $T_{2}$}

$$
\begin{aligned}
T_{2} & =\mathcal{E}_{\hat{\mathbf{h}}}\left\{z^{2} \hat{\mathbf{h}}^{H}\left(\alpha^{2} P \mathbf{Q}+\sigma^{2} \mathbf{I}\right) \hat{\mathbf{h}}\right\}= \\
& =\mathcal{E}_{\hat{\mathbf{h}}}\left\{z^{2} \hat{\mathbf{h}}^{H}\left(\alpha^{2} P \boldsymbol{\Theta}^{H} \mathbf{S}_{\mathbf{Q}} \boldsymbol{\Theta}+\sigma^{2} \boldsymbol{\Theta}^{H} \boldsymbol{\Theta}\right) \hat{\mathbf{h}}\right\}= \\
& =\mathcal{E}_{\hat{\mathbf{h}}}\left\{z^{2} \hat{\mathbf{h}}^{H} \boldsymbol{\Theta}^{H}\left(\alpha^{2} P \mathbf{S}_{\mathbf{Q}}+\sigma^{2} \mathbf{I}\right) \boldsymbol{\Theta} \hat{\mathbf{h}}\right\}= \\
& =\mathcal{E}_{\mathbf{v}}\left\{z^{2} \mathbf{v}^{H}\left(\alpha^{2} P \mathbf{S}_{\mathbf{Q}}+\sigma^{2} \mathbf{I}\right) \mathbf{v}\right\}= \\
& =\mathcal{E}_{\mathbf{v}}\left\{z^{2} \mathbf{v}^{H} \mathbf{S}_{\mathbf{M}} \mathbf{v}\right\}
\end{aligned}
$$

where $\mathbf{S}_{\mathbf{M}}=\alpha^{2} P \mathbf{S}_{\mathbf{Q}}+\sigma^{2} \mathbf{I}$ is a diagonal matrix with diagonal elements $\mathbf{S}_{\mathbf{M} i i}=\alpha^{2} P \mathbf{S}_{\mathbf{Q}_{i i}}+\sigma^{2}$. Substituting $z$ :

$$
\begin{aligned}
T_{2} & =\frac{1}{\alpha^{2} P} \mathcal{E}_{\mathbf{v}}\left\{\int_{x=0}^{\infty} x e^{-x\left(\mathbf{v}^{H} \mathbf{v}+\frac{\sigma^{2}}{\alpha^{2} P}\right)} d x \mathbf{v}^{H} \mathbf{S}_{\mathbf{M} \mathbf{v}}\right\}= \\
& =\frac{1}{\alpha^{2} P} \mathcal{E}_{\mathbf{v}}\left\{\int_{x=0}^{\infty} x e^{-x\left(\mathbf{v}^{H} \mathbf{v}\right)} e^{-x \frac{\sigma^{2}}{\alpha^{2} P}} d x \mathbf{v}^{H} \mathbf{S}_{\mathbf{M}} \mathbf{v}\right\}= \\
& =\frac{1}{\alpha^{2} P} \int_{x=0}^{\infty} x e^{-x \frac{\sigma^{2}}{\alpha^{2} P}} \mathcal{E}_{\mathbf{v}}\left\{\prod_{i} e^{-x v_{i}^{H} v_{i}} \sum_{k} v_{k}^{H} \mathbf{S}_{\mathbf{M} k k} v_{k}\right\} d x .
\end{aligned}
$$

Similarly to the derivation of the expectation expression in $T_{1}$, the expectation in (12) can be written as:

$$
\begin{gathered}
\mathcal{E}_{\mathbf{v}}\left\{\prod_{i} e^{-x\left(v_{i}^{H} v_{i}\right)} \sum_{k} v_{k}^{H} \mathbf{S}_{\mathbf{M} k k} v_{k}\right\}= \\
=\sum_{k} \mathbf{S}_{\mathbf{M} k k} \mathcal{E}_{v_{k}}\left\{\left|v_{k}\right|^{2} e^{-x\left|v_{k}\right|^{2}}\right\} \prod_{i \neq k} \mathcal{E}_{v_{i}}\left\{e^{-x\left|v_{i}\right|^{2}}\right\}= \\
=\sum_{k} \mathbf{S}_{\mathbf{M} k k} \frac{r_{k}}{\left(x+r_{k}\right)^{2}} \prod_{i \neq k} \frac{r_{i}}{x+r_{i}}= \\
=\sum_{k} \mathbf{S}_{\mathbf{M} k k} \frac{1}{x+r_{k}} \prod_{i} \frac{r_{i}}{x+r_{i}} .
\end{gathered}
$$

Finally $T_{2}$ is

$$
T_{2}=\frac{1}{\alpha^{2} P} \sum_{k} \mathbf{S}_{\mathbf{M} k k} \int_{x=0}^{\infty} x e^{-x \frac{\sigma^{2}}{\alpha^{2} P}} \frac{1}{x+r_{k}} \prod_{i} \frac{r_{i}}{x+r_{i}} d x .
$$

E. Term $T_{3}$

$$
\begin{aligned}
T_{3} & =2 \alpha \sqrt{P} \mathcal{E}_{\hat{\mathbf{h}}}\left\{z \operatorname{Re}\left\{\hat{\mathbf{h}}^{H} \mathbf{D} \hat{\mathbf{h}}\right\}\right\}= \\
& =2 \alpha \sqrt{P} \mathcal{E}_{\hat{\mathbf{h}}}\left\{z \operatorname{Re}\left\{\hat{\mathbf{h}}^{H} \boldsymbol{\Theta}^{H} \mathbf{S}_{\mathbf{D}} \boldsymbol{\Theta} \hat{\mathbf{h}}\right\}\right\}= \\
& =2 \alpha \sqrt{P} \mathcal{E}_{\mathbf{v}}\left\{z \operatorname{Re}\{\underbrace{\mathbf{v}^{H} \mathbf{S}_{\mathbf{D}} \mathbf{v}}_{\text {real }}\}=2 \alpha \sqrt{P} \mathcal{E}_{\mathbf{v}}\left\{z \mathbf{v}^{H} \mathbf{S}_{\mathbf{D}} \mathbf{v}\right\}\right\} .
\end{aligned}
$$

and, for the expectation, the same approach is applicable as above. That is:

$$
T_{3}=2 \alpha \sqrt{P} \frac{1}{\alpha \sqrt{P}} \mathcal{E}_{\mathbf{v}}\left\{\int_{x=0}^{\infty} e^{-x\left(\mathbf{v}^{H} \mathbf{v}+\frac{\sigma^{2}}{\alpha^{2} P}\right)} d x \mathbf{v}^{H} \mathbf{S}_{\mathbf{D}} \mathbf{v}\right\}=
$$




$$
\begin{aligned}
& =2 \mathcal{E}_{\mathbf{v}}\left\{\int_{x=0}^{\infty} e^{-x\left(\mathbf{v}^{H} \mathbf{v}\right)} e^{-x \frac{\sigma^{2}}{\alpha^{2} P}} d x \mathbf{v}^{H} \mathbf{S}_{\mathbf{D}} \mathbf{v}\right\}= \\
= & 2 \int_{x=0}^{\infty} e^{-x \frac{\sigma^{2}}{\alpha^{2} P}} \mathcal{E}_{\mathbf{v}}\left\{\prod_{i} e^{-x v_{i}^{H} v_{i}} \sum_{k} v_{k}^{H} \mathbf{S}_{\mathbf{D} k k} v_{k}\right\} d x= \\
= & 2 \int_{x=0}^{\infty} e^{-x \frac{\sigma^{2}}{\alpha^{2} P}} \sum_{k} \mathbf{S}_{\mathbf{D} k k} \frac{1}{x+r_{k}} \prod_{i} \frac{r_{i}}{x+r_{i}} d x= \\
= & 2 \sum_{k} \mathbf{S}_{\mathbf{D} k k} \int_{x=0}^{\infty} e^{-x \frac{\sigma^{2}}{\alpha^{2} P}} \frac{1}{x+r_{k}} \prod_{i} \frac{r_{i}}{x+r_{i}} d x .
\end{aligned}
$$

\section{REFERENCES}

[1] T. Kim and J. G. Andrews, "Optimal Pilot-to-Data Power Ratio for MIMO-OFDM,” IEEE Globecom, 2005.

[2] — - "Balancing Pilot and Data Power for Adaptive MIMO-OFDM Systems," IEEE Globecom, 2005.

[3] E. Golovins and N. Ventura, "Optimal Training for the SM-MIMOOFDM Systems with MMSE Channel Estimation," 6th Annual Communication Networks and Services Research Conference, pp. 470-477, 2008.

[4] V. K. V. Gottumukkala and H. Minn, "Capacity Analysis and Pilot-Data Power Allocation for MIMO-OFDM With Transmitter and Receiver IQ Inbalances and Residual Carrier Frequency Offset," IEEE Trans. Veh. Techn., pp. 553-565, 2012.

[5] G. Fodor and M. Telek, "On the pilot-data power trade off in single input multiple output systems," Royal Institute of Technology KTH, Stockholm, Sweden, Tech. Rep. http://urn.kb.se/resolve?urn=urn:nbn:se:kth:diva-139408, January 2011.

[6] P. Soldati, M. Johansson, G. Fodor, and S. Sorrentino, "On Pilot Dimensioning in Multicell Single Input Multiple Output Systems," IEEE Workshop on Broadband Wireless Access, Houston, TX, USA,2011.

[7] R. Chen, J. G. Andrews, R. W. Heath, and A. Ghosh, "Uplink Power Control in Multi-cell Spatial Multiplexing Wireless Systems," IEEE Trans. on Wireless Communications, vol. 6, no. 7, pp. 2700-2711, July 2007.

[8] T. Marzetta, "Noncooperative cellular wireless with unlimited numbers of base station antennas," IEEE Trans. Wireless Comm., vol. 9, no. 11, pp. 3590-3600, 2010.

[9] S. M. Kay, Fundamentals of Statistical Signal Processing, Vol. I: Estimation Theory. Prentice Hall, 1993, no. ISBN: 0133457117.

[10] R. Gallager, "Circularly-Symmetric Gaussian Complex Vectors," http://www.rle.mit.edu/rgallager/documents/CircSymGauss.pdf, 2008.

[11] H. Yin, D. Gesbert, M. Filippou, and Y. Liu, "A coordinated approach to channel estimation in large-scale multiple-antenna systems," http://arxiv.org/abs/1203.5924, no. arXiv:1203.5924v1 [cs.IT], March 2012.

[12] I. Gradshteyn and I. Ryzhik, Table of Integrals, Series and Products. Academic Press, 2007, no. ISBN-13: 978-0-12-373637-6. 